\title{
Assessment of Chlamydia trachomatis infection by Cobas Amplicor PCR and in-house LightCycler assays using PreservCyt and 2-SP media in voluntary legal abortions
}

\author{
Correspondence \\ Farida Hamdad \\ hamdadfarida2002@yahoo.fr
}

Received 27 January 2008

Accepted 26 August 2008

\author{
Henri Sevestre, ${ }^{1}$ Jacques Mention, ${ }^{2}$ Jean-François Lefebvre, ${ }^{3}$ \\ François $\mathrm{Eb}^{4}$ and Farida Hamdad ${ }^{4}$ \\ ${ }^{1}$ Laboratoire d'Anatomo-Pathologie, Centre Hospitalo-Universitaire d'Amiens, Amiens, France \\ ${ }^{2}$ Centre de Gynécologie-Obstétrique, Centre Hospitalo-Universitaire d'Amiens, Amiens, France \\ ${ }^{3}$ Centre de Ressources Régionales en Biologie Moléculaire, Université de Picardie Jules Verne, \\ Amiens, France \\ ${ }^{4}$ Service de Bactériologie, Centre Hospitalo-Universitaire d'Amiens, Amiens, France
}

Chlamydial infection of the upper genital tract after abortion is well recognized, but routine screening for infection before termination is rare, and few centres are aware of the prevalence of post-abortion complications in their patient population. Knowledge of the patient population is the best guide for developing screening strategies. The aim of this study was to determine the prevalence of chlamydial infection in patients presenting for legal termination of pregnancy, and to assess the presence of Chlamydia trachomatis by PCR on specimens collected in either PreservCyt (ThinPrep) or 2-sucrose phosphate (2-SP) transport medium. Two hundred and eleven single, sexually active women, aged 15-26 years, attending the Gynaecology and Obstetric Hospital, Amiens, France, for surgical termination of pregnancy were enrolled in this study from June 2002 to June 2003. C. trachomatis detection using a Cobas Amplicor PCR test (Roche Diagnostics) targeting a 207 bp segment of the common cryptic plasmid and a quantitative LightCycler real-time PCR (LC-PCR) (Roche Diagnostics) targeting a $123 \mathrm{bp}$ fragment within the highly conserved constant domain 3 of the single-chromosome-copy omp $A$ gene were performed on endocervical swabs in 2-SP, and on specimens collected using a cytobrush and placed in PreservCyt medium. The in-house LC-PCR was used as a chromosomal diagnosis method and to determine the load of $C$. trachomatis. This method was able to detect the mutant Swedish variant with a deletion of $377 \mathrm{bp}$ in the target area in the cryptic plasmid, which is the region targeted by the Cobas Amplicor PCR test. C. trachomatis was detected in 19/211 patients ( $9 \%$ ) by both PCR methods. Among the 19 infected women, C. trachomatis was detected by the Cobas Amplicor PCR in 16 specimens in PreservCyt (7.6\%) and in 12 endocervical swabs in 2-SP (5.7\%). Specimens from only nine women were PCR-positive in both PreservCyt and 2-SP media by this method. Cobas Amplicor PCR revealed that 10.9 and $2.3 \%$ of the PreservCyt and 2-SP samples, respectively, contained inhibitors. The same 19 infected women were LC-PCR positive in both PreservCyt and 2-SP samples. No additional infected women were found by this last method; thus, it was concluded that none of the samples contained

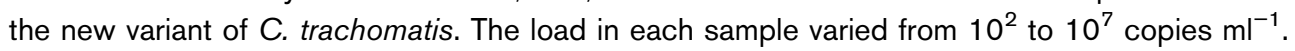

\section{INTRODUCTION}

Chlamydia trachomatis is the most common bacterial sexually transmitted infection (STI) and is capable of

Abbreviations: CDC, Centers for Disease Control and Prevention; HPV, human papillomavirus; IC, internal control; LC-PCR, LightCycler real-time PCR; PID, pelvic inflammatory disease; STI, sexually transmitted infection. causing infection with serious complications. Current sexual practices favouring transmission of $C$. trachomatis include early age of first intercourse, poor condom use and an overall increase in the number of life-time sexual partners. Generally, the public does not perceive itself to be at great risk, probably owing to the frequently asymptomatic nature of chlamydial infection. Seventy per cent of infections in women are asymptomatic and therefore 
untreated (Cates \& Wasserheit, 1991; Hamdad et al., 2004), and a significant proportion remains undiagnosed, allowing the development of complications such as pelvic inflammatory disease (PID) (Scholes et al., 1996; AdderleyKelly \& Stephens, 2005). A total of $20 \%$ of women who develop PID become infertile, $18 \%$ develop chronic pelvic pain and $9 \%$ have a tubal pregnancy (CDC, 2002).

Screening and treatment are particularly important for women at risk of upper genital tract complication, such as those who undergo a genital tract surgical procedure, for example termination of pregnancy. Indeed, C. trachomatis is the leading aetiological agent for PID among sexually active young women who have undergone induced abortion (Groom et al., 2001). Accordingly, women terminating their pregnancy represent an appropriate population for sentinel surveillance of chlamydial infection, particularly because of their young age, the high proportion of single women and the relative inconsistent use of contraception.

Therefore, a patient population presenting for legal surgical termination of pregnancy in our hospital was screened for C. trachomatis and human papillomavirus (HPV) and monitored for precancerous lesions of the cervix. PreservCyt solution (ThinPrep) was used to preserve cervical samples before the preparation of ThinPrep slides, and as medium for the collection and transport of specimens for both C. trachomatis and HPV detection by nucleic acid tests.

The performance of the PreservCyt transport medium was compared with the classical 2-sucrose phosphate (2-SP) transport medium for $C$. trachomatis detection by nucleic acid amplification tests. Two methods were used: a Cobas Amplicor PCR test and an in-house quantitative real-time LightCycler PCR (LC-PCR) (Roche Diagnostics) assay targeting a $123 \mathrm{bp}$ fragment within the highly conserved constant domain 3 of the single-chromosome-copy ompA gene. A hybrid capture-based system (Digene) was used for HPV screening. HPV results will be reported separately.

\section{METHODS}

Preliminary assay. A major problem with nucleic acid amplification tests is the presence of potential inhibitors that can lead to falsenegative results. Therefore, the aim of the preliminary study was to assess the presence of endogenous amplification inhibitors and to determine the technical feasibility of using PreservCyt, which contains a methanol-based fixative, as a storage medium (ThinPrep Pap Test; Cytyc). This liquid medium preserves organisms and DNA but may inhibit PCR.

Technical feasibility was determined by testing tenfold serial dilutions of a suspension of C. trachomatis serovar E (DK-20) in PreservCyt medium to yield $10000,1000,100,10$ or 1 inclusion-forming units $\mathrm{ml}^{-1}$. The testing of $100 \mu \mathrm{l}$ each dilution for DNA extraction and amplification with a Cobas Amplicor PCR test according to the manufacturer's protocol for cervical swabs showed that the medium inhibited the PCR. Therefore, these dilutions were centrifuged at $13000 \mathrm{~g}$ for $30 \mathrm{~min}$. The supernatant was discarded and DNA extraction was performed on the cell pellet according to the manufacturer's protocol for urine. Using this protocol, all dilutions were positive for the Cobas Amplicor PCR and the internal control (IC) was detected. These results led us to compare C. trachomatis detection in clinical samples in PreservCyt and 2-SP transport media.

Patients. Two hundred and eleven single patients who attended the Gynaecologic and Obstetric Hospital, Amiens, France, for surgical termination of pregnancy between June 2002 and June 2003 were invited to take part. The patient's age and duration of gestation were noted.

Written informed consent was obtained from all participants. All patients were asked to give an address where they could be contacted confidentially for any necessary treatment.

Specimens. An endocervical specimen from each woman was collected by standard procedures for $C$. trachomatis detection using an endocervical swab, and the contents were transferred immediately into a tube containing $2 \mathrm{ml}$ 2-SP transport medium ( $0.2 \mathrm{M}$ sucrose/ $0.02 \mathrm{M}$ phosphate; Quelab Laboratories). Samples were sent to the hospital's Department of Medical Microbiology.

Specimens were also collected using a cytobrush. The brush head was detached and placed in a vial of PreservCyt transport medium. The PreservCyt specimens were sent to the hospital's Cytology and Clinical Pathology Department. After the cytology slide had been prepared and read satisfactorily, an HPV test was performed $(\mathrm{H}$. Sevestre, personal communication). One millilitre of these specimens was then sent to the Department of Medical Microbiology within $24 \mathrm{~h}$ of collection for C. trachomatis detection using PCR. The order of the specimens was randomized throughout the study.

\section{Amplification tests.}

Endocervical swabs in 2-SP and specimens in PreservCyt medium (211 of each) were tested using a C. trachomatis Cobas Amplicor PCR test and an in-house LC-PCR assay. Standard precautions were used to prevent cross-contamination.

Cobas Amplicor PCR test. The primers targeted a 207 bp segment of the common cryptic plasmid, which is used as target DNA for the Cobas Amplicor PCR test. The cryptic plasmid is identical in all serovars of $C$. trachomatis and approximately ten copies of the plasmid are present in every organism. Hence, using the plasmid as target DNA increases the sensitivity of the system. The Cobas Amplicor CT/NG PCR test was performed according to the manufacturer's instructions.

The tubes of specimens in PreservCyt medium were vortexed vigorously and centrifuged at $13000 \mathrm{~g}$ for $30 \mathrm{~min}$. The supernatant was discarded and the manufacturer's DNA extraction protocol for urine was used on the cell pellet. The lysate tubes were placed in a heating block at $95{ }^{\circ} \mathrm{C}$ for $10 \mathrm{~min}$ to inactivate cervical inhibitors (Verkooyen et al., 1996; Hamdad-Daoudi et al., 2004).

The endocervical swab specimens collected in 2-SP medium were processed as follows: $1 \mathrm{ml}$ each endocervical specimen was centrifuged at $13000 \mathrm{~g}$ for $30 \mathrm{~min}$, as for the specimens in PreservCyt medium. The manufacturer's DNA extraction protocol for cervical swabs was used on the cell pellet and the lysate tubes were incubated at $95{ }^{\circ} \mathrm{C}$ for $10 \mathrm{~min}$.

A total of $50 \mu \mathrm{l}$ each extracted nucleic acid was added to $50 \mu \mathrm{l}$ Cobas Amplicor master mix and amplified according to the manufacturer's instructions. An IC to detect inhibitors in the sample causing a falsenegative result was incorporated in the master mix. Amplification and detection of C. trachomatis and IC DNA were performed automatically by the Cobas Amplicor system. AmpErase uracil- $N$-glycosylase was incorporated to prevent carryover contamination. Specimens 
were considered positive or negative in accordance with the manufacturer's guidelines.

Quantitative real-time LC-PCR. Eighty microlitres of Cobas Amplicor preparation were processed using a QIAamp DNA mini kit (Qiagen), according to the manufacturer's instructions, to further purify and concentrate the DNA sample. Quality control for DNA extraction was performed by inclusion of a C. trachomatis culture genotype F (positive control), and nuclease-free water (Promega) was used as a negative control.

The in-house real-time quantitative PCR assays were performed using a LightCycler 1.0 (Roche Diagnostics). Primer sequences (CT1 and CT2) were used following the SYBR Green I LC-PCR format as described by Solomon et al. (2003). The LightCycler DNA Master SYBR Green I used includes dUTP to prevent carryover contamination.

The preparation of standards for quantification of chlamydial organisms was performed as described by Solomon et al. (2003). The SYBR Green I assay was performed on amplicons generated from serial dilutions of genomic DNA prepared from pure elementary bodies of $C$. trachomatis genotype $\mathrm{F}$. This assay included seven concentrations of standard (tenfold dilutions of the suspension from $10^{6}$ to 1 copy per capillary tube) and a negative control; these were used to generate a standard curve. The amount of chlamydial DNA was calculated using this standard curve. To study intra-assay variation, each dilution was performed with four replicates.

Real-time PCR was performed in glass capillary tubes and the total reaction volume per tube was $20 \mu$. Each capillary tube was loaded with $4 \mu \mathrm{l}$ LightCycler FastStart reaction/enzyme mix SYBR Green (final concentration $1 \times$ ) (Roche Diagnostics), $1 \mu \mathrm{l} 0.5 \mathrm{pM}$ each primer, and $5 \mu \mathrm{l}$ purified DNA from standard and clinical specimens, and made up to $20 \mu \mathrm{l}$ with nuclease-free water. A negative control, in which the $5 \mu \mathrm{l}$ DNA was replaced with nuclease-free water, was included in each run. Because the small volume $(5 \mu \mathrm{l})$ increases the effect of variability between samples with different DNA amounts, we tried to compensate for this by doing two replicate assays per sample. The capillary tubes were closed, centrifuged at $735 \mathrm{~g}$ for $30 \mathrm{~s}$ in a microcentrifuge using the LightCycler centrifuge adapters and placed in the LightCycler sample carousel, which was inserted into the LightCycler instrument for sample amplification.

The FastStart Taq DNA polymerase was activated by heating at $95{ }^{\circ} \mathrm{C}$ for $10 \mathrm{~min}$. This denaturation was followed by 45 amplification cycles of denaturation at $95{ }^{\circ} \mathrm{C}$ for $10 \mathrm{~s}$, annealing at $60{ }^{\circ} \mathrm{C}$ for $15 \mathrm{~s}$ and elongation at $72{ }^{\circ} \mathrm{C}$ for $15 \mathrm{~s}$, followed by a single measurement of the SYBR Green I fluorescence signal. The melting curve $\left(T_{\mathrm{m}}\right)$ analysis was performed for 10 min immediately following completion of the PCR. Thus, samples were heated to $95{ }^{\circ} \mathrm{C}$ (at $20{ }^{\circ} \mathrm{C} \mathrm{s}^{-1}$ ) without hold, cooled to $60{ }^{\circ} \mathrm{C}$ (at $20{ }^{\circ} \mathrm{C} \mathrm{s}^{-1}$ ), held for $15 \mathrm{~s}$, heated slowly at $0.1{ }^{\circ} \mathrm{C} \mathrm{s}^{-1}$ up to $95{ }^{\circ} \mathrm{C}$ and finally cooled to $40{ }^{\circ} \mathrm{C}$ (at $20^{\circ} \mathrm{C} \mathrm{s}^{-1}$ ) for $30 \mathrm{~s}$.

A melting-curve step was included to confirm the amplification. Thus, the presence or absence of specific PCR products was determined by the $T_{\mathrm{m}}$ analysis, which can differentiate non-specific products from the specific product (the $T_{\mathrm{m}}$ of non-specific products is lower). Only those specimens that gave a peak that was clearly distinguishable from the primer dimer (negative control) were considered positive. Analysis of PCR amplification curves and melting curves was carried out using LightCycler software version 3.5. A calculation of C. trachomatis copy number was taken at the crossing point of each sample during the exponential phase of amplification.

\section{RESULTS AND DISCUSSION}

A total of 211 patients was invited to take part in the study. The mean age was 21 years (interquartile range 15-26), 54
$(25.6 \%)$ were less than 20 years old, $140(66.3 \%)$ were 20 24 years old and $17(8.0 \%)$ were $25-26$ years old. All reported no current regular sexual partner. The duration of gestation when the pregnancy was interrupted was known: $183(86.7 \%)$ were at $8-12$ weeks' gestation and $28(13.2 \%)$ were at 13 weeks' gestation.

C. trachomatis DNA was found in $19(9.0 \%)$ of the women by both PCR methods. The prevalence of chlamydial infection varied significantly with age (Table 1 ). Among the 19 infected women, C. trachomatis was detected by Cobas Amplicor PCR in 16 specimens (7.6\%) in PreservCyt medium and in 12 specimens $(5.7 \%)$ in 2-SP medium. Nine specimens $(4.3 \%)$ were positive for Cobas Amplicor PCR in both PreservCyt and 2-SP, seven (3.3\%) were positive in PreservCyt alone and three specimens (1.4\%) were negative in PreservCyt but positive in 2-SP medium. The PreservCyt and 2-SP results were concordant in $82 \%$ patients.

Initially, the IC Cobas Amplicor PCR revealed that 23 $(10.9 \%)$ of the PreservCyt specimens and $5(2.3 \%)$ of the specimens that were collected in the 2-SP medium contained inhibitors. The labile inhibitors in cervical mucus can be inactivated by heating to $95^{\circ} \mathrm{C}$; thus, the inhibited samples in 2-SP were retested after freezing, thawing and heating at $95{ }^{\circ} \mathrm{C}$ for $10 \mathrm{~min}$. The inhibited samples in PreservCyt, which contains a methanol-based fixative that may inhibit PCR, were retested after a $1: 5$ dilution of the lysate together with heating at $95{ }^{\circ} \mathrm{C}$ for $10 \mathrm{~min}$. Despite these treatments, five specimens $(2.3 \%)$ in PreservCyt medium still contained inhibitors. None of the inhibitory specimens became $C$. trachomatis-positive after retesting.

The sensitivity of Cobas Amplicor PCR in PreservCyt and 2-SP samples was 84.21 and $63.15 \%$, respectively. The lower sensitivity of Cobas Amplicor PCR, particularly in 2SP medium, may have been related to low levels of $C$. trachomatis DNA being present.

All samples positive by Cobas Amplicor PCR either in PreservCyt or 2-SP were positive by the LC-PCR assay in

Table 1. The age distribution of patients and prevalence of $C$. trachomatis infection

\begin{tabular}{|lcc|}
\hline Age (years) & $\begin{array}{c}\text { No. of patients } \\
(\%)\end{array}$ & $\begin{array}{c}\text { No. positive in 2-SP } \\
\text { or PreservCyt by either } \\
\text { Cobas Amplicor PCR } \\
\text { or LC-PCR (\%) }\end{array}$ \\
\hline $15-16$ & $3(1.4)$ & $0(0)$ \\
$17-18$ & $22(10.4)$ & $1(4.5)$ \\
$19-20$ & $56(26.5)$ & $7(12.5)$ \\
$21-22$ & $73(34.6)$ & $7(9.6)$ \\
$23-24$ & $39(18.5)$ & $2(5.1)$ \\
$25-26$ & $18(8.5)$ & $2(11.1)$ \\
Total & 211 & $19(9)$ \\
\hline
\end{tabular}


both PreservCyt and 2-SP media. Among the nine specimens that were positive by Cobas Amplicor PCR in both PreservCyt and 2 SP, seven were LC-PCR-positive in the two replicate samples. Among the ten specimens that were positive by Cobas Amplicor PCR either in PreservCyt or 2SP medium, one was LC-PCR-positive in the two replicate samples. The other 11 specimens were LC-PCR-positive in only one of the replicates. The variation in results may be associated with the low copy numbers of $C$. trachomatis. Indeed, the intra-assay variation of the serial fourfold dilution of the genomic DNA from cultured C. trachomatis genotype $\mathrm{F}$ showed a variation in results when one copy per capillary tube was present (data not shown).

The specificity of the LC-PCR assay was improved by determining the $T_{\mathrm{m}}$ of the specific PCR product and the primer dimers. Melting-curve analysis of the ompA PCR products of the serial dilution from cultured C. trachomatis showed that the template concentration did not affect the $T_{\mathrm{m}}$.

The $T_{\mathrm{m}}$ of C. trachomatis amplicons in the SYBR Green LC-PCR was determined to be $85{ }^{\circ} \mathrm{C}\left( \pm 2{ }^{\circ} \mathrm{C}\right)$ and was confirmed by running $10 \mu \mathrm{l}$ each product on an ethidium bromide-stained $4 \%$ agarose gel (data not shown). The $T_{\mathrm{m}}$ of C. trachomatis was clearly distinguishable from the $T_{\mathrm{m}}$ of the primer dimers, which was below $80{ }^{\circ} \mathrm{C}$ (Fig. 1).

Scatter plots of the estimated number of copies of ompA $\mathrm{ml}^{-1}$ in each sample (analysis was performed using
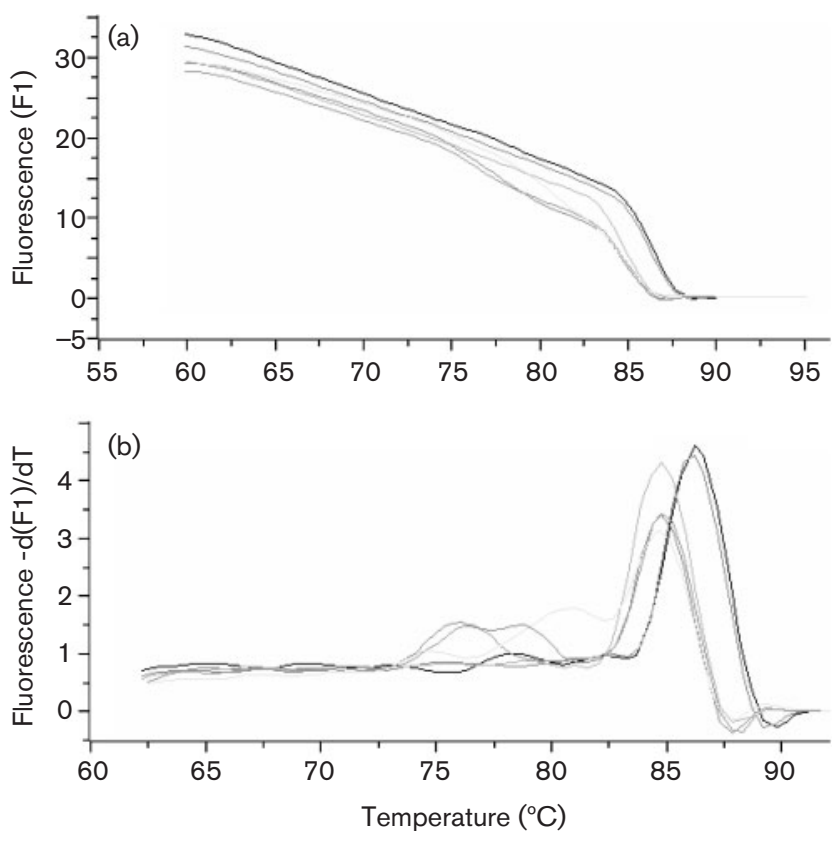

Fig. 1. Melting analysis of the C. trachomatis ompA PCR assay for three endocervical samples (each run was performed in duplicate). (a) Melting curve, (b) melting peaks of the specific ompA products $\left(T_{\mathrm{m}}=85^{\circ} \mathrm{C} \pm 2{ }^{\circ} \mathrm{C}\right)$ and primer dimers $\left(T_{\mathrm{m}}=74-81^{\circ} \mathrm{C}\right)$. second-derivation methods) are shown in Fig. 2. We obtained this number by multiplication of the copy number per capillary tube measured by quantitative PCR, taking into account the total volume used. Most women had a rather low chlamydial load (below 1000 genome copies $\mathrm{ml}^{-1}$ ) and there was no association between age and chlamydial load, as described by Gomes et al. (2006). Each patient with a positive diagnosis of $C$. trachomatis infection received $1 \mathrm{~g}$ azithromycin orally in a single dose.

Nine per cent of the women had low-grade squamous intraepithelial lesions. C. trachomatis and HPV co-infection was documented in eight women $(3.8 \%)$ between 19 and 22 years of age.

Young age is one of the most important risk factors for STIs and, as a direct result, women can develop PID (Simms et al., 2006), which is a primary cause of infertility. C. trachomatis is one of the major causes of tubal infertility, and at least $10 \%$ of women who have a single episode of pelvic infection will become subfertile (Westrom, 1987; Cates et al., 1990; Hamdad et al., 2004).

The presence of lower genital tract infection increases the risk of complications, and diagnosis of chlamydial infection and treatment are necessary to prevent these complications and to control the spread of infection. As most $C$. trachomatis infections are asymptomatic, there is a need to screen high-risk patients to reduce the morbidity from infection, as well as to decrease the incidence and prevalence of this pathogen in the population at large (Scholes et al., 1996; Groom et al., 2001). It is important to emphasize that the highest prevalence of chlamydial infections, recurrences and complications occurs in sexually active young women. These should have the highest priority for screening, but few physicians offer such screening.

Women requesting abortion are at significant risk of harbouring STIs. Indeed, preoperative cervical screening of 211 women undergoing first-trimester induced surgical

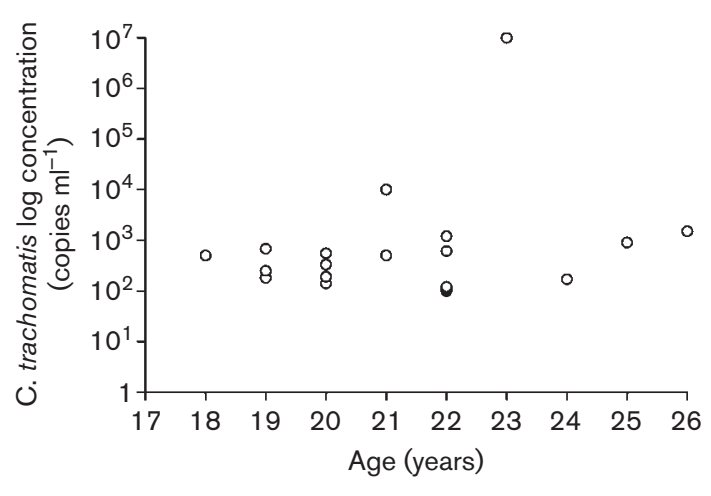

Fig. 2. Quantification of $C$. trachomatis in endocervical samples by real-time PCR: estimated number of copies of ompA ml-1 according to age. 
abortions in Centre Hospitalo-Universitaire d'Amiens (Amiens University Hospital) yielded C. trachomatis in $9.0 \%$ of patients. This rate is consistent with previous studies that reported the highest risk of exposure to chlamydial infection among this population (Blackwell et al., 1993; Cameron et al., 2003; de Barbeyrac et al., 2006), but is higher than in other studies (Garland et al., 2000; Groom et al., 2001; Mallinson et al., 2002).

The highest prevalence was noted in women between 19 and 22 years of age $(14 / 129 ; 10.8 \%)$, followed by women up to 23 years of age $(4 / 57 ; 7.0 \%)$. In contrast, although previous research (Mertz et al., 2001; Adderley-Kelly \& Stephens, 2005) has consistently identified the highest rates of STIs among younger adolescents (aged 15-19), our older participants ( $>19$ years) had the highest proportion of STIs.

Pelvic infection is the most common complication of legal abortions, and the majority of women wishing to disrupt their pregnancy in our study population were young $(99.5 \%$ were aged $\leqslant 25)$. It is therefore important for maintaining fertility that the abortion procedure be safe and without serious complications. In this study, none of the infected and treated women developed complications.

PreservCyt could be used as an alternative medium for the collection and transport of specimens for the detection of C. trachomatis, as described in published studies (Anguenot et al., 2001; Bianchi et al., 2002; Zhang et al., 2002; Koumans et al., 2003; Hopwood et al., 2004; Keegan et al., 2005; Chernesky et al., 2007), but specimens must be processed after the centrifugation step as described in this and other studies (Bianchi et al., 2002; Koumans et al., 2003). In addition, the cell pellet must be washed twice with a buffer before DNA extraction, as described by Keegan et al. (2005). Indeed, one major problem encountered during this study was the increased occurrence of inhibition of the amplification reaction. The optimization of the method of DNA extraction from PreservCyt is essential to avoid inhibitors and to ensure adequate sensitivity of detection (Keegan et al., 2005).

The cellular quantity of the sample significantly affected the ability to detect Chlamydia by Cobas Amplicor PCR. Indeed, in this study, $5.7 \%$ of infected patients yielded positive results for endocervical swab specimens in 2-SP, whilst $7.6 \%$ of infected subjects yielded positive results in PreservCyt medium. When comparing endocervical swab samples in 2-SP with specimens in PreservCyt, we found that $3.3 \%$ of endocervical swab samples were negative, an indication of poorly collected specimens.

The sensitivity of the Cobas Amplicor PCR was affected by the clinical sample, whilst the LC-PCR assay was not. The LC-PCR assay detected more positive specimens than the Cobas Amplicor PCR in both PreservCyt (9 vs 7.6\%) and 2-SP (9 vs $5.7 \%$ ) but no more infected women. The concentration of purified DNA, the fluorescence detection steps and using two replicate assays per sample increased the sensitivity of the LC-PCR.
The in-house LC-PCR assay was used as a diagnostic method and to determine the relative yield of $C$. trachomatis as copy number. This method targeting a chromosomal fragment had the advantage of being able to detect the new Swedish variant with a deletion of $377 \mathrm{bp}$ in the target area in the cryptic plasmid (Ripa \& Nilsson, 2007), which was the region targeted by the Cobas Amplicor PCR, and confirmed that none of these samples contained the new variant.

C. trachomatis and HPV co-infection was detected in eight women $(3.8 \%)$; as co-infections may precipitate precancerous lesions, these patients should be followed carefully. This study provides additional evidence that a single cervical specimen can be used for cervical cytology and screening for C. trachomatis using nucleic acid amplification tests. The advantages of a single specimen include the need for only one collection device, the preservation of cellular material and nucleic acid in one liquid medium, and the ability to detect not only C. trachomatis but other vaginal or cervical infections as well (Fiel-Gan et al., 1999; Inhorn et al., 2001). Thus, the patient does not have to be examined repeatedly and there is no need for multiple specimen collection kits or different procedures for specimen processing (Koumans et al., 2003). In addition, the possibility of conserving cervical samples in liquid medium at room temperature could facilitate the procedure in screening programmes (Bianchi et al., 2002; Koumans et al., 2003; Hopwood et al., 2004; Keegan et al., 2005).

This study also shows that the development of real-time PCR methods has elevated clinical nucleic acid amplification testing to a new level. Assay turnaround time can now be as short as $1 \mathrm{~h}$ because of simultaneous product amplification and detection. Real-time PCR is a fast and effective method for the detection and quantification of $C$. trachomatis load in clinical samples.

Voluntary legal abortion is an excellent opportunity to provide information to the patient, and to screen and treat STIs. A limited number of studies in this population has been published (Blackwell et al., 1993; Garland et al., 2000; Groom et al., 2001; Mallinson et al., 2002; Cameron et al., 2003; LaMontagne et al., 2004; de Barbeyrac et al., 2006). The current screen-and-treat policy has, however, been shown to be defective in several regions and should become more common as legal abortion becomes more frequent. We urge all doctors who carry out the termination of pregnancies to assess the prevalence of chlamydial infection, and to consider the costs and benefits of screening and prophylaxis for chlamydial infection.

Public health-based screening programmes in France are underfunded and offer screening to less than half of the target population. In common with other screening programmes (for cancerous lesions), screening for STIs targeted to 'high-risk' populations would decrease the prevalence of Chlamydia infection and reduce the incidence of PID. 


\section{ACKNOWLEDGEMENTS}

This article is dedicated to the memory of Professor Jeanne Orfila. Our admiration and deep recognition for the rigour of her scientific reasoning and the knowledge that she transmitted to us until the end of her life is a lasting legacy. We thank Patrik Bavoil for critical review of the manuscript.

\section{REFERENCES}

Adderley-Kelly, B. \& Stephens, E. M. (2005). Chlamydia: a major health threat to adolescents and young adults. ABNF J 16, 52-55.

Anguenot, J. L., de Marval, F., Vassilakos, P., Auckenthaler, R., Ibéchéole, V. \& Campana, A. (2001). Combined screening for Chlamydia trachomatis and squamous intra-epithelial lesions using a single liquid-based cervical sample. Hum Reprod 16, 2206-2210.

Bianchi, A., Moret, F., Desrues, J. M., Champenois, T., Dervaux, Y., Desvouas, O., Oursin, A., Quinzat, D., Dachez, R. \& other authors (2002). PreservCyt transport medium used for the Thin Prep Pap Test is a suitable medium for detection of Chlamydia trachomatis by the Cobas Amplicor CT/NG test: results of a preliminary study and future implications. J Clin Microbiol 40, 1749-1754.

Blackwell, A. L., Thomas, P. D., Wareham, K. \& Emery, S. J. (1993). Health gains from screening for infection of the lower genital tract in women attending for termination of pregnancy. Lancet 342, 206-210.

Cameron, S. T., Stewart, S. \& Sutherland, S. (2003). Can a busy abortion service cope with a screen-and-treat policy for Chlamydia trachomatis infection? Int J STD AIDS 14, 50-54.

Cates, W. \& Wasserheit, J. N. (1991). Genital chlamydial infections: epidemiology and reproductive sequelae. Am J Obstet Gynecol 164, 1771-1781.

Cates, W., Rolfs, R. T. \& Aral, S. O. (1990). Sexually transmitted diseases, pelvic inflammatory disease, and infertility: an epidemiologic update. Epidemiol Rev 12, 199-220.

CDC (2002). Sexually transmitted diseases treatment guidelines 2002. MMWR Recomm Rep 51, 1-78.

Chernesky, M., Freund, G. G., Hook, E., Leone, P., D’Ascoli, P. \& Martens, M. (2007). Detection of Chlamydia trachomatis and Neisseria gonorrhoeae infections in North American women by testing SurePath liquid-based Pap specimens in APTIMA assays. J Clin Microbiol 45, 2434-2438.

de Barbeyrac, B., Tilatti, K., Raherison, S., Mathieu, C., FrantzBlancpain, S., Clerc, M., Goulet, V., Bébéar, C. \& Hocké, C. (2006). Screening of Chlamydia trachomatis infection in a family planning center and a birth control clinic, Bordeaux, France, 2005 BEH 37-38, 277-279.

Fiel-Gan, M. D., Villamil, C. F., Mandavilli, S. R., Ludwig, M. E. \& Tsongalis, G. J. (1999). Rapid detection of HSV from cytologic specimens collected into ThinPrep fixative. Acta Cytol 43, 1034-1038.

Garland, S. M., Tabrizi, S., Hallo, J. \& Chen, S. (2000). Assessment of Chlamydia trachomatis prevalence by PCR and LCR in women presenting for termination of pregnancy. Sex Transm Infect 76, 173176.

Gomes, J. P., Borrego, M. J., Atik, B., Santo, I., Azevedo, J., Brito de Sa, A., Nogueira, P. \& Dean, D. (2006). Correlating Chlamydia trachomatis infectious load with urogenital ecological success and disease pathogenesis. Microbes Infect 8, 16-26.

Groom, T. M., Stewart, P., Kruger, H. \& Bell, G. (2001). The value of a screen and treat policy for Chlamydia trachomatis in women attending for termination of pregnancy. J Fam Plann Reprod Health Care 27, 69-72.
Hamdad, F., Orfila, J., Boulanger, J. C. \& Eb, F. (2004). Chlamydia trachomatis urogenital infection in women. Best diagnosis approaches. Gynecol Obstet Fertil 32, 1064-1074.

Hamdad-Daoudi, F., Petit, J. \& Eb, F. (2004). Assessment of Chlamydia trachomatis infection in asymptomatic male partners of infertile couples. J Med Microbiol 53, 985-990.

Hopwood, J., Mallinson, H., Hodgson, E. \& Hull, L. (2004). Liquid based cytology: examination of its potential in a chlamydia screening programme. Sex Transm Infect 80, 371-373.

Inhorn, S. L., Wand, P. J., Wright, T. C., Hatch, K. D., Hallum, J. \& Lentrichia, B. B. (2001). Chlamydia trachomatis and Pap testing from a single, fluid-based sample. A multicenter study. J Reprod Med 46, 237-242.

Keegan, H., Boland, C., Malkin, A., Griffin, M., Ryan, F. \& Lambkin, H. (2005). Comparison of DNA extraction from cervical cells collected in PreservCyt solution for the amplification of Chlamydia trachomatis. Cytopathology 16, 82-87.

Koumans, E. H., Black, C. M., Markowitz, L. E., Unger, E. R., Pierce, A., Sawyer, M. K. \& Papp, J. R. (2003). Comparison of methods for detection of Chlamydia trachomatis and Neisseria gonorrhoeae using commercially available nucleic acid amplification tests and a liquid pap smear medium. J Clin Microbiol 41, 1507-1511.

LaMontagne, D. S., Pimenta, J. M., Fenton, K. A., Mallison, H. \& Howood, J. (2004). Management of genital chlamydial infections at termination of pregnancy services in England and Wales: where are we now? BJOG 111, 1408-1412.

Mallinson, H., Hopwood, J., Skidmore, S., Frenton, K., Phillips, C. \& Jones, I. (2002). Provision of chlamydia testing in a nationwide service offering termination of pregnancy: with data capture to monitor prevalence of infection. Sex Transm Infect 78, 416-421.

Mertz, K. J., Ransom, R. L., St Louis, M. E., Groseclose, S. L., Hadgu, A., Levine, W. C. \& Hayman, C. (2001). Prevalence of genital chlamydial infection in young women entering a national job training program, 1990-1997. Am J Public Health 91, 1287-1290.

Ripa, T. \& Nilsson, P. A. (2007). A Chlamydia trachomatis strain with a 377-bp deletion in the cryptic plasmid causing false-negative nucleic acid amplification tests. Sex Transm Dis 34, 255-256.

Scholes, D., Stergachis, A., Heidrich, F. E., Andrilla, H., Holmes, K. K. \& Stamm, W. E. (1996). Prevention of pelvic inflammatory disease by screening for cervical chlamydial infection. $N$ Engl J Med 23, 1362 1366. Medline

Simms, I., Stephenson, J. M., Mallison, H., Peeling, R. W., Thomas, K., Gokhale, R., Rogers, P. A., Hay, P., Oakeshott, P. \& other authors (2006). Risk factors associated with pelvic inflammatory disease. Sex Transm Infect 82, 452-457.

Solomon, A. W., Holland, M. J., Burton, M. J., West, S. K., Alexander, N. D., Aguirre, A., Massae, P. A., Mkocha, H., Munoz, B. \& other authors (2003). Strategies for control of trachoma: observational study with quantitative PCR. Lancet 362, 198-204.

Verkooyen, R. P., Luijendijk, A., Huisman, W. M., Goessens, W. H., Kluytmans, J. A., Van Rijsoort-Vos, J. H. \& Verbrugh, H. A. (1996). Detection of PCR inhibitors in cervical specimens by using the AMPLICOR Chlamydia trachomatis assay. J Clin Microbiol 34, 30723074.

Westrom, L. (1987). Pelvic inflammatory disease: bacteriology and sequelae. Contraception 36, 111-128.

Zhang, W., Cohenford, M., Lentrichia, B., Isenberg, H. D., Simson, E., Li, H., Yi, J. \& David, Y. (2002). Detection of Chlamydia trachomatis by isothermal ramification amplification method: a feasibility study. $J$ Clin Microbiol 40, 128-132. 